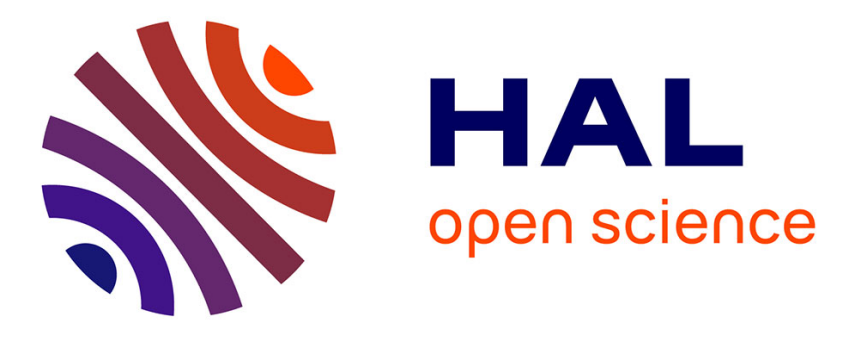

\title{
Ultrafast imaging of in vivo muscle contraction using ultrasound
}

Thomas Deffieux, Jean-Luc Gennisson, Mickaël Tanter, Mathias Fink, Antoine Nordez

\section{- To cite this version:}

Thomas Deffieux, Jean-Luc Gennisson, Mickaël Tanter, Mathias Fink, Antoine Nordez. Ultrafast imaging of in vivo muscle contraction using ultrasound. Applied Physics Letters, 2006, 89 (18), pp.184107. 10.1063/1.2378616 . hal-02481829

\section{HAL Id: hal-02481829 \\ https://hal.science/hal-02481829}

Submitted on 17 Feb 2020

HAL is a multi-disciplinary open access archive for the deposit and dissemination of scientific research documents, whether they are published or not. The documents may come from teaching and research institutions in France or abroad, or from public or private research centers.
L'archive ouverte pluridisciplinaire HAL, est destinée au dépôt et à la diffusion de documents scientifiques de niveau recherche, publiés ou non, émanant des établissements d'enseignement et de recherche français ou étrangers, des laboratoires publics ou privés. 


\section{Ultrafast imaging of in vivo muscle contraction using ultrasound}

Cite as: Appl. Phys. Lett. 89, 184107 (2006); https://doi.org/10.1063/1.2378616

Submitted: 07 July 2006 . Accepted: 21 September 2006. Published Online: 03 November 2006

Thomas Deffieux, Jean-Luc Gennisson, Mickaël Tanter, Mathias Fink, and Antoine Nordez

\section{ARTICLES YOU MAY BE INTERESTED IN}

Explososcan: A parallel processing technique for high speed ultrasound imaging with linear phased arrays

The Journal of the Acoustical Society of America 75, 1273 (1984); https://

doi.org/10.1121/1.390734

Sono-activated ultrasound localization microscopy

Applied Physics Letters 103, 174107 (2013); https://doi.org/10.1063/1.4826597

Adaptive focusing in scattering media through sound-speed inhomogeneities: The van Cittert Zernike approach and focusing criterion

The Journal of the Acoustical Society of America 96, 3721 (1994); https://

doi.org/10.1121/1.410562

Lock-in Amplifiers Find out more today

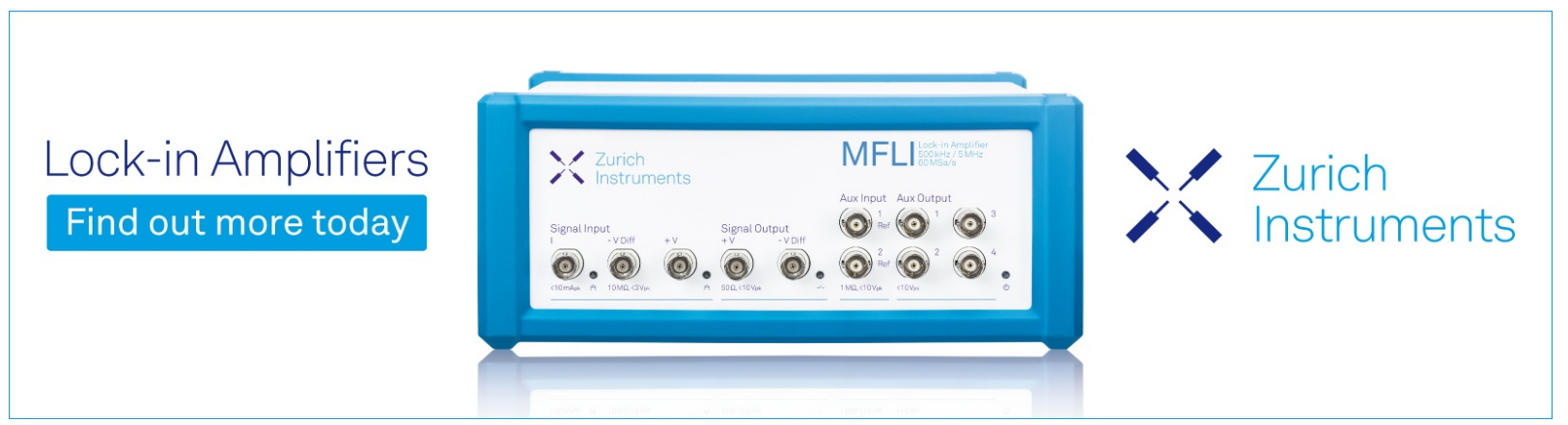




\title{
Ultrafast imaging of in vivo muscle contraction using ultrasound
}

\author{
Thomas Deffieux, ${ }^{\text {a) }}$ Jean-Luc Gennisson, Mickaël Tanter, and Mathias Fink \\ Laboratoire Ondes et Acoustique, ESPCI, Université Paris VII, CNRS UMR 7587, 10 Rue Vauquelin 75005 \\ Paris, France

\begin{abstract}
Antoine Nordez
Laboratoire "Motricité, Interactions, Performance," JE 2438, UFR STAPS, Université de Nantes, Nantes Atlantique Universités, 25 bis Bd. Guy Mollet BP 72206, Nantes F-44000, France
\end{abstract}

(Received 7 July 2006; accepted 21 September 2006; published online 3 November 2006)

\begin{abstract}
In this letter, an innovative way of imaging transient and local shear vibrations of an in vivo contracting muscle is proposed. The principle is to use an ultrafast ultrasound scanner (up to 5000 frames s$^{-1}$ ) able to follow with a submillimeter resolution the motion of the muscle tissue in a two dimensional plane. This ultrafast echographic imaging technique leads to both local and transient in vivo studies of the contraction of a muscle as reported by these first experiments done on the biceps brachii. (C) 2006 American Institute of Physics. [DOI: 10.1063/1.2378616]
\end{abstract}

Numerous monitoring techniques are commonly used to study muscular or neuromuscular function. Electromyography (EMG) is used to record the electrical activity of the muscle. It can be reported as the sum of action potentials propagating in a muscle's fibers. ${ }^{1}$ Mechanomyography is the recording of the muscular vibrations produced by the active muscle. It can be used as a monitor of muscle stiffness and could be related to the muscle force production. ${ }^{2}$ Unfortunately, all of these methods have a poor accuracy to assess local measurements and are thus not suitable for fully understanding the underlying structure and mechanical behavior of the muscle. In order to create maps of the local response of the muscle, a few techniques have been applied to reconstruct the local velocity distributions of the muscle in three or two dimensions (3D or 2D): phase-contrast magneticresonance imaging can reconstruct full 3D images of the muscle motion in a stroboscopic way. From these images local strains are calculated. ${ }^{3}$ Doppler tissue imaging gives the tissues' velocity distribution in a $2 \mathrm{D}$ plane and allows axial strain assessments. ${ }^{4}$ Recently, ultrasound image correlations at low frame rates have also been used to track the muscle motion. ${ }^{5}$ While very promising, these techniques can only image the muscle up to a few tens of frames per second. These low frame rates cannot be considered high enough to fully visualize the transient phenomena occurring during muscle activation.

Recently, ultrafast ultrasound scanners were designed by our group. Our last generation of echographic devices gives access to $2 \mathrm{D}$ radio frequency (rf) images at a few thousand hertz using a modified imaging sequence, i.e., a hundred times faster than any conventional ultrasound scanner. From these rf images, $B$-mode images are constructed. The cross correlation between two successive images permits us to assess the local axial particle velocity, and a complete movie of the axial velocity maps can be finally deduced. Such an approach allows us to provide both very high spatial (submillimetric) and temporal accuracy (less than a millisecond sampling), overcoming all the respective drawbacks of the previously cited techniques. This scanner ${ }^{6}$ is a 128 multichannel fully programmable system driving any kind of con-

\footnotetext{
${ }^{a)}$ Electronic mail: thomas.deffieux @espci.fr
}

ventional ultrasonic probe. The sequence consists in emitting a single plane wave pulse. Ultrasonic echoes backscattered by tissue heterogeneities are then stored in memories. These ultrasonic raw data are processed in a posttreatment to create images with submillimetric resolution by applying a conventional beam formation (time delay and sum operations) in the receive mode. Finally, consecutive echographic images are compared using one dimensional cross correlation along the ultrasound beam axis in order to compute their axial relative displacements ${ }^{7}$ (note that "axial" terminology refers to the ultrasonic beam $z$ direction and not to the fibers' main axis, see Fig. 1). This method enables us to measure relative displacements as low as $1 \mu \mathrm{m}$ between two consecutive frames and thus gives an estimation of tissue local velocity.

To trigger the muscle contraction with the imaging system, surface neuromuscular electrostimulation was achieved. A homemade electrostimulation device composed of a function generator (Agilent, model 33220A, Palo Alto, CA, USA), a power amplifier (Brüel \& Kjær, type 2718, Nærum, Denmark), and two noninvasive surface electrodes (Compex, Ecublens, Switzerland) were used. The cathode was placed on the motor point, localized by qualitatively looking for the maximum muscle excitation, and the anode was placed on the distal portion of the biceps. A $30 \mathrm{~V}$ amplitude, $400 \mu \mathrm{s}$

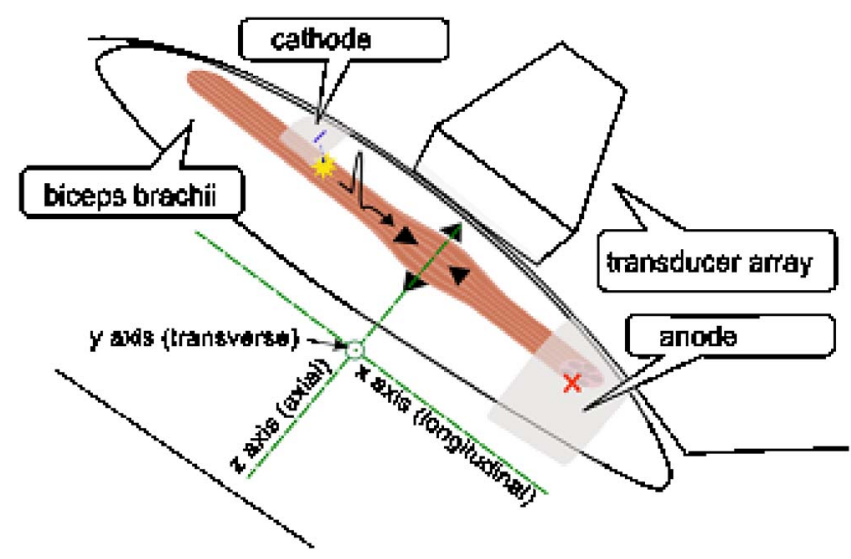

FIG. 1. (Color online) Measurement protocol. Muscle fibers were excited with neuromuscular electrostimulation on the motor point, and the axial displacements were acquired with the $2 \mathrm{D}$ ultrasound ultrafast scanner. Both the arm and the transducer array were strongly fixed to avoid movements. 


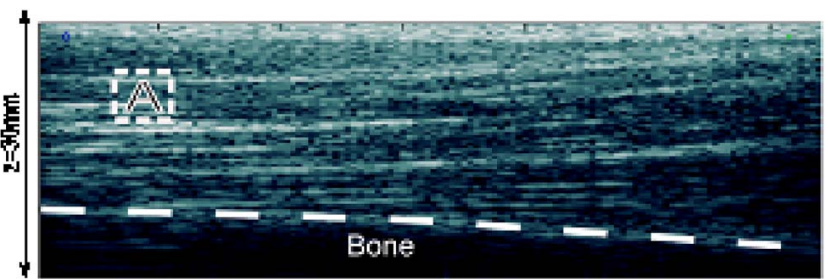

(a)

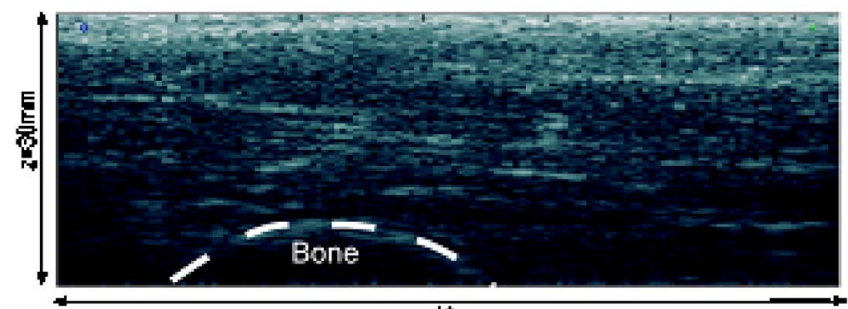

(b)

x=\$ínm

FIG. 2. (Color online) B-mode images of the muscle. Probe was either parallel (a) or perpendicular (b) to the fibers. The aligned fibers of the biceps brachii are clearly visible.

wide rectangular pulse was used to fire an action potential in the fibers just below the motor point (Fig. 1).

In our first experiments, a conventional $8 \mathrm{MHz}$ transducer array was placed either parallel (longitudinal position) or perpendicular (transverse position) to the fibers' main direction in order to follow the contraction in different planes (Fig. 1). This means that the displacements seen were never those corresponding to the shortening of the fibers but rather those corresponding to their lateral widening.

After the acquisition of a conventional gray-scale echographic image (Fig. 2), the scanner took 500 images at $1500 \mathrm{~Hz}$, and a movie of the axial velocity (Fig. 3) was computed. This movie was used to retrieve contraction and relaxation times and create maps of the maximum axial displacement. The average tissue velocity $(\mathrm{cm} / \mathrm{s})$ in the in situ region of interest A (Fig. 2) is plotted as a function of time (Fig. 4). Contraction time is taken as the positive part of the axial velocity (fiber bundles contracting and thus moving toward the probe), while relaxation time is the negative part (fiber bundles moving back to its original position). Contraction time is thus found to be around $58 \mathrm{~ms}$ and relaxation time around $120 \mathrm{~ms}$, values that are consistent with the literature. ${ }^{8}$ The axial ( $z$ direction) displacements can be computed from the integration of the axial tissue velocity. De-

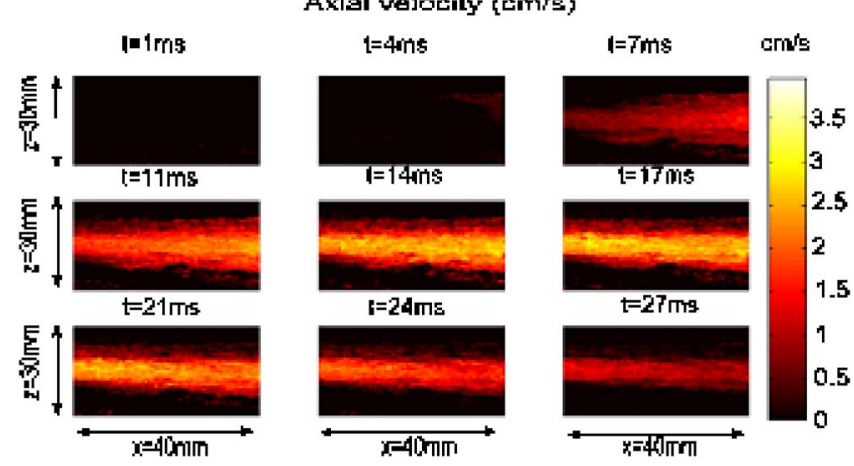

FIG. 3. (Color online) Movie of the axial velocity (cm/s). A $30 \mathrm{~V}$ amplitude, $400 \mu$ s wide square pulse excitation on the motor point triggers the contraction. The contracting area can easily be visualized. The ultrasonic probe is placed parallel to the fibers; the imaging frame rate is set to $1500 \mathrm{~Hz}$.

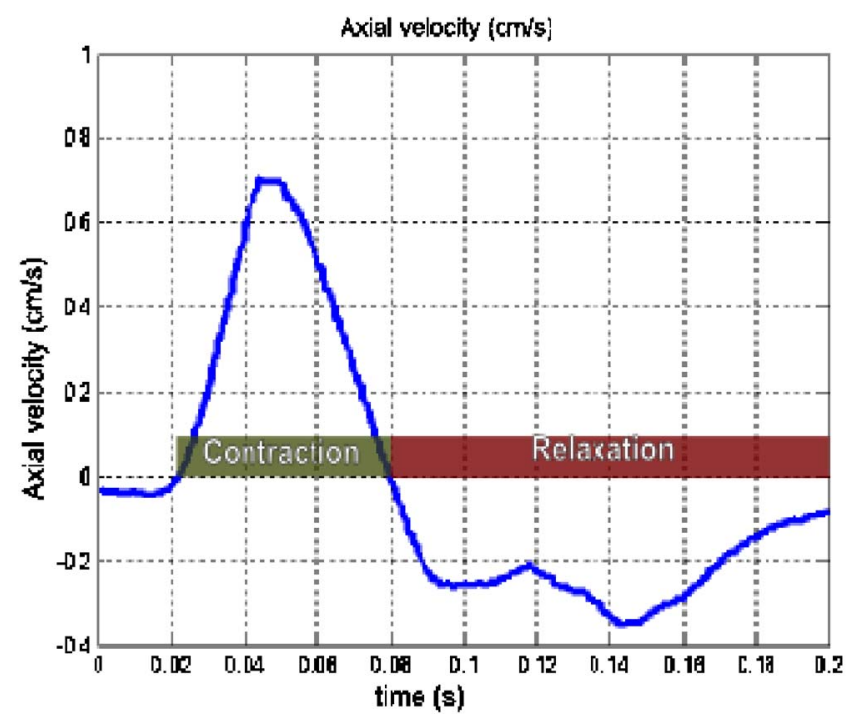

FIG. 4. (Color online) Axial velocity profile $(\mathrm{cm} / \mathrm{s})$ vs time $(\mathrm{s})$ in region of interest A (Fig. 2).

tecting the maximum local displacements over time enables us to create maps of the contracting fiber bundles (Fig. 5).

To illustrate the temporal accuracy of the scanner, periodic excitations with different pulse repetition rates (from 5 to $20 \mathrm{~Hz}$ with $5 \mathrm{~Hz}$ steps) have been used and displacements computed inside a region of interest A (Fig. 2). Depending on the frequency of the excitation and as expected from surface EMG measurements but now for each point inside the muscle, temporal summation and tetany can be observed (Fig. 6). As the frequency of excitation rises, muscle fibers do not have the time to come back to their initial position and temporal summation occurs, leading to a stronger contraction. ${ }^{1}$ When the sum of the contraction and relaxation times is less than the period of stimulation pulse, no temporal summation occurs $(5 \mathrm{~Hz})$. When the contraction time is less but the sum of the contraction and relaxation times is greater, oscillations can be seen $(10$ and $15 \mathrm{~Hz})$. Finally, when the contraction time is greater than the period of excitation, the displacement is an increasing curve, reaching a maximum $(20 \mathrm{~Hz})$.

The ultrafast echographic imaging approach enables the assessment of the transient mechanical behavior of entire muscles with a millimetric resolution. From the acquisition of local axial tissue velocities of tissues over the entire organ, one can compute not only axial displacements but also axial strains by simply deriving the displacement field along

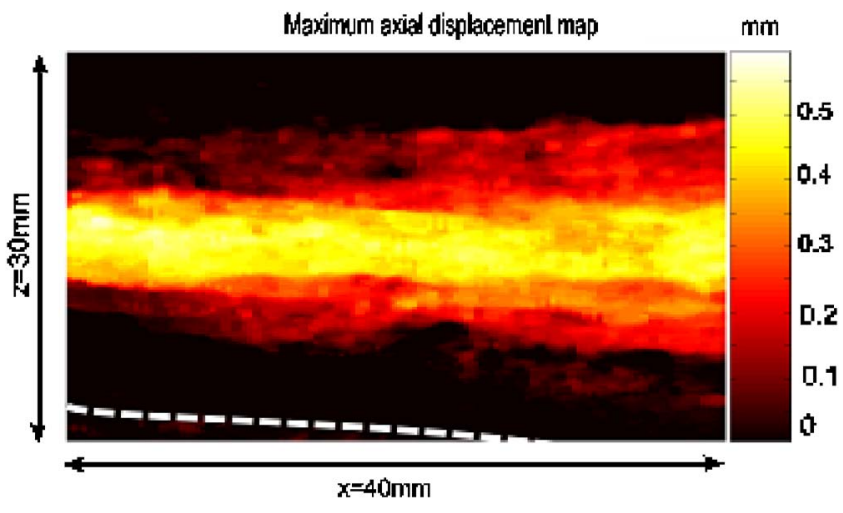

FIG. 5. (Color online) Maximum axial displacement map (mm). 


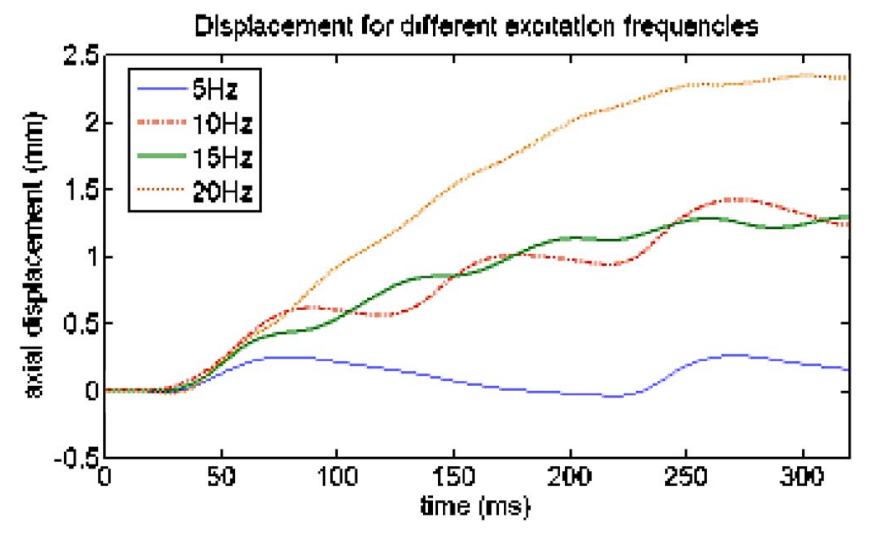

FIG. 6. (Color online) Displacement $(\mathrm{mm})$ vs time $(\mathrm{ms})$ in a region of interest for different excitation frequencies.

the $z$ direction in a posttreatment step. It allows us to get rid of the global translational movements of the muscle. However, this operation is always sensitive to noise and do not discriminate active and passive movements.

Much beyond the visualization of strains, the spatial and temporal accuracies of the system will allow us to retrieve the exact localization of the contracting bundles. This requires us to recover the active displacement sources by solving the inverse source problem of the elastic wave propagation. Assuming the medium to be linear, homogeneous, isotropic, incompressible, and piecewise homogeneous, and considering only the null divergence terms (shear components), the elastodynamics equation ${ }^{9}$ describing the elastic wave propagation can be described as follows:

$$
\rho \frac{\partial^{2} \mathbf{u}}{\partial t^{2}}(\mathbf{r}, t)=\mu \Delta \mathbf{u}(\mathbf{r}, t)+\mathbf{s}(\mathbf{r}, t),
$$

where $\rho, \mu, \mathbf{u}(\mathbf{r}, t)$, and $\mathbf{s}(\mathbf{r}, t)$ represent density, Young's modulus, displacement fields, and the volumetric force source terms, respectively. Equation (1) requires the knowledge of Young's modulus (stiffness) as well as the longitudinal ( $x$ axis, along the fibers) and transverse ( $y$ axis, perpendicular to the imaging plane) displacements. Shear modulus has already been measured in the biceps brachii ${ }^{10,11}$ and also shown to be transverse isotropic along fibers. ${ }^{10}$ Transverse displacements can thus be assumed to have the same order of magnitude as the measured axial ( $z$ axis) displacements. Moreover, longitudinal displacements along fibers could be computed using a synthetic aperture technique ${ }^{12}$ already implemented on ultrafast echographic devices. Using this complementary information, the only remaining unknown is $\mathbf{s}(\mathbf{r}, t)$. The inverse source problem can be solved, permitting us to retrieve the localization of the transient sources of contraction inducing the global contraction of the muscle.

Using this ultrafast device, a high space and time resolution imaging of a transient in vivo muscle contraction was achieved. We were able to follow the contraction of the muscle and to extract both local and transient properties such as the contraction time, the relaxation time, or maps of the contracting areas. The ultrafast echographic scanner, coupled with an EMG device, will provide a powerful tool to understand the link between electrical and mechanical activities of the muscle and to study the spatial recruitment of fibers and its associated disorders such as fatigue and neuromuscular diseases.

${ }^{1}$ J. V. Basmajian and C. De Luca, Muscles Alive (Williams and Wilkins, Baltimore, 1983), Vol. 1.

${ }^{2}$ T. W. Beck, T. J. Housh, J. T. Cramer, J. P. Weir, G. O. Johnson, J. W. Coburn, M. H. Malek, and M. Mielke, Biomed. Eng. Online 4, 67 (2005).

${ }^{3}$ J. E. Drace and N. J. Pelc, Radiology 193, 423 (1994).

${ }^{4}$ N. R. Grubb, A. Fleming, G. R. Sutherland, and K. A. Fox, Radiology 194, 837 (1995)

${ }^{5}$ I. D. Loram, C. N. Maganaris, and M. Lakie, J. Appl. Physiol. 100, 1311 (2006).

${ }^{6}$ L. Sandrin, M. Tanter, S. Catheline, and M. Fink, IEEE Trans. Ultrason. Ferroelectr. Freq. Control 49, 426 (2002).

${ }^{7}$ J. Ophir, I. Céspedes, H. Ponnekanti, Y. Yasdi, and X. Li, Ultrason. Imaging 13, 111 (1991).

${ }^{8}$ F. Buchtal and H. Schmalbruch, Acta Physiol. Scand. 79, 435 (1970).

${ }^{9}$ L. M. Brekhovskikh and V. Goncharov, Mechanics of Continua and Wave Dynamics (Springer, Berlin, 1993), Vol. 1.

${ }^{10}$ J. L. Gennisson, S. Catheline, S. Chaffaï, and M. Fink, J. Acoust. Soc. Am. 114, 536 (2003).

${ }^{11}$ J. Bercoff, M. Tanter, and M. Fink, IEEE Trans. Ultrason. Ferroelectr. Freq. Control 51, 396 (2004).

${ }^{12}$ M. Tanter, J. Bercoff, L. Sandrin, and M. Fink, IEEE Trans. Ultrason. Ferroelectr. Freq. Control 49, 1363 (2002). 